\title{
Weak Poincaré inequalities and hitting times for jump processes
}

\author{
Huihui Cheng ${ }^{1 *}$ and Hongde Xiao ${ }^{2}$
}

"Correspondence:

chenghuihui@ncwu.edu.cn

${ }^{1}$ College of Mathematics and Information Sciences, North China

University of Water Resources and Electric Power, Zhengzhou, 450045,

P.R. China

Full list of author information is

available at the end of the article

\begin{abstract}
In this paper, we get a criteria of weak Poincaré inequality by some integrability of hitting times for jump processes. In fact, integrability of hitting times on a subset $F$ of state space $E$ implies that the taboo process restricted on $E \backslash F$ is decay, from which we get a weak Poincaré inequality with absorbing (Dirichlet) boundary. Using it and a local Poincaré inequality, we obtain a weak Poincaré inequality by the decomposition method.
\end{abstract}

Keywords: weak Poincaré inequality; hitting times; jump process

\section{Introduction and main results}

During the recent years, a lot of progress has been made in the understanding of functional inequalities and their links with the convergence rates of Markov processes. As we know, the convergence rates of a Markov semigroup and the corresponding functional inequalities can be determined by each other. To describe the convergence rates slower than exponential, Röckner and Wang [1] introduced the following weak Poincaré inequality (WPI):

$$
\operatorname{Var}_{\mu}(f) \leq \alpha(r) D(f, f)+r \Phi(f), \quad f \in \mathcal{D}(D), r>0
$$

where $\operatorname{Var}_{\mu}(f)=\|f-\mu(f)\|_{2}^{2}, \alpha$ is a nonnegative decreasing function on $(0, \infty), D(f, f)=$ $-\mu(f L f), L$ is the generator of a Markov semigroup $P_{t}$ on $L^{2}(\mu)$, and $\Phi: L^{2}(\mu) \rightarrow(0, \infty)$ satisfies $\Phi(c f)=c^{2} \Phi(f)$ and $\Phi\left(P_{t} f\right) \leq \Phi(f)$ for any $c \in \mathbb{R}$ and $f \in L^{2}(\mu)$. They proved that the $L^{2}$-convergence rate of a Markov semigroup and the corresponding weak Poincaré inequality can be determined by each other. We restate the results as follows. Let $\left(P_{t}\right)_{t \geq 0}$ be the Markov semigroup determined by the Dirichlet form $(D, \mathcal{D}(D))$. Assume that (1.1) holds. Then

$$
\left\|P_{t} f-\mu(f)\right\|_{2}^{2} \leq \xi(t)\left[\Phi(f)+\|f\|_{2}^{2}\right], \quad t>0,
$$

where $\xi(t)=\inf \left\{r>0:-\frac{1}{2} \alpha(r) \log (r) \leq t\right\}$. Conversely, in the reversible case, (1.2) implies the weak Poincaré inequality (1.1) for $\alpha(r)=2 r \inf _{s>0} \frac{1}{s} \xi^{-1}(s \exp [1-s / r])$. In this paper, we choose $\Phi(\cdot)=\|\cdot\|_{L^{\infty}(\mu)}^{2}=:\|\cdot\|_{\infty}^{2}$.

Recently, much literature was devoted to the relationships of the Poincaré-type inequalities and Lyapunov conditions used in the 'Meyn-Tweedie' theory (see [2, 3]). Cattlaux

(c) 2016 Cheng and Xiao. This article is distributed under the terms of the Creative Commons Attribution 4.0 International License (http://creativecommons.org/licenses/by/4.0/), which permits unrestricted use, distribution, and reproduction in any medium, provided you give appropriate credit to the original author(s) and the source, provide a link to the Creative Commons license, and indicate if changes were made. 
et al. [4] went a step further by showing the equivalence between the (usual) Poincaré inequality, Lyapunov conditions, and the existence of exponential moments for hitting times for reversible diffusion processes. Now it is interesting to look at more general moments of hitting times and other processes. Mao and Xia [5] obtained a criterion for spectral gap by hitting times for jump processes via decomposition method. Cheng and Wang [6] studied the algebraic convergence rates for diffusion processes on Riemannian manifolds with boundary by using a Lyapunov condition. In the present paper, we use some integrability of hitting times to get a type of weak Poincaré inequality for jump processes, which can be used to study the convergence rates for jump processes in the sense of $\left\|P_{t}-\pi\right\|_{\infty \rightarrow 2}$.

Let $(q(x), q(x, d y))$ be the $q$-pair of a regular reversible $q$-process with transition kernel $P_{t}(x, d y)$ on a probability space $(E, \mathscr{E}, \mu)$. Denote by $\mathscr{E}_{b}$ the set of bounded functions on $\mathscr{E}$. For $f \in{ }_{b} \mathscr{E}$, denote by $L$ and $P_{t}$ the generator and semigroup, respectively, where $L f(x)=\int_{E} q(x, d y)(f(y)-f(x))$, and $P_{t} f(x)=\int_{E} f(y) P_{t}(x, d y)$. The Dirichlet form $D$ is defined by $D(f, f)=\frac{1}{2} \int_{E \times E} \mu(d x) q(x, d y)(f(y)-f(x))^{2}$, where $f \in \mathcal{D}(D)=\left\{f \in L^{2}(\mu): D(f, f)<\infty\right\}$. Now, we introduce two inequalities used in this paper.

Taking $F \in \mathscr{E}$ such that $\mu(F)>0$, we have the inequality

$$
\mu\left(f^{2}\right) \leq \alpha_{F}(r) D(f, f)+r\|f\|_{\infty}^{2}, \quad f \in \mathcal{D}(D),\left.f\right|_{F}=0, r>0,
$$

which is said to be a weak Poincaré inequality with Dirichlet boundary (WPID).

A local Poincaré inequality (LPI) restricted on $F$ is satisfied if

$$
\operatorname{Var}_{\mu_{F}}(f) \leq C D_{F}(f, f), \quad f \in \mathcal{D}\left(D_{F}\right)
$$

where $D_{F}(f, f)=\frac{1}{2} \int_{F \times F} \mu_{F}(d x) q(x, d y)(f(y)-f(x))^{2}$ and $\mu_{F}(\cdot)=\frac{\mu(\cdot \cap F)}{\mu(F)}$. Define

$$
\lambda_{1}(F)=\inf \left\{D_{F}(f, f): \mu_{F}(f)=0, \mu_{F}\left(f^{2}\right)=1\right\} .
$$

Then $\lambda_{1}(F)^{-1}$ is the smallest constant such that local Poincaré inequality restricted on $F$ holds.

This paper is organized as follows. In Section 2, we prove that some integrability of hitting times on a subset of state space is sufficient for the WPID (1.3). In Section 3, we show how to use LPI and WPID to get WPI.

The following is the main result of this paper.

Theorem 1.1 Fix $F \in \mathscr{E}$ with $\mu(F)>0$. Assume that a local Poincaré inequality restricted on $F$ is satisfied for a reversible q-processes with q-pair $(q(x), q(x, d y))$. Let $\tau_{F}:=\inf \{t \geq 0$ : $\left.X_{t} \in F\right\}$ be the hitting time of $F$. If there exist a decreasing function $\xi:[0, \infty) \rightarrow(0, \infty)$ such that $\xi(t) \rightarrow 0$ as $t \rightarrow \infty, \mathbb{E}_{\mu} \xi\left(\tau_{F}\right)^{-1}=: c<\infty$, and $M_{F}:=\sup _{x \in F} q\left(x, F^{c}\right)<\infty$, then we have the weak Poincaré inequality

$$
\operatorname{Var}_{\mu}(f) \leq \alpha(r) D(f, f)+r\|f\|_{\infty}^{2}, \quad f \in \mathcal{D}(D), r>0,
$$

for $\alpha(r) \leq\left(1+\frac{M_{F}}{\lambda_{1}(F)}\right) \xi^{-1}\left(\frac{r}{4 c}\right)+\frac{1}{\lambda_{1}(F)}$.

In this theorem, we get a criterion of weak Poincaré inequality by integrability of hitting times and a local Poincaré inequality on a fixed subset for jump processes. Since the subset is fixed, it is different from the criterion of WPI in Theorem 4.3.1 of [7]. 


\section{The hitting time and weak Poincaré inequality with Dirichlet boundary}

In this section, we prove that some integrability of hitting times on a subset $F$ implies that the taboo process restricted on $E \backslash F$ is decay, by which we get a weak Poincaré inequality with Dirichlet boundary.

Denote ${ }_{F} P_{t}(x, H)=\mathbb{P}_{x}\left[X_{t} \in H, t<\tau_{F}\right]$. Then ${ }_{F} P_{t}(x, d y)$ satisfies the backward Kolmogorov equation (see [8])

$$
{ }_{F} P_{t}(x, H)=\int_{0}^{t} e^{-q(x)(t-s)} \int \frac{q(x, d y \backslash F)}{q(x)}{ }_{F} P_{s}(y, H) d s+e^{-q(x) t} 1_{F}(x), \quad x \in F^{c} .
$$

So the $q$-pair of ${ }_{F} P_{t}(x, d y)$ is $\left({ }_{F} q(x),{ }_{F} q(x, d y)\right)$ with ${ }_{F} q(x, H)=q(x, H \backslash F)$ and ${ }_{F} q(x)=q(x)$ for $x \in F^{c}$ and 0 otherwise; ${ }_{F} P_{t}$ is a Markov semigroup,

$$
{ }_{F} P_{t} f(x)=\int_{F^{c}} P_{t}(x, d y) f(y), \quad f \in L^{2}\left(F^{c}, \mu\right)
$$

Its generator ${ }_{F} L$ is defined as

$$
{ }_{F} L f(x)=\int_{F^{c}} q(x, d y) f(y)-q(x) f(x), \quad f \in L^{2}\left(F^{c}, \mu\right) .
$$

Here $(\cdot, \cdot)$ denotes the inner product in $L^{2}\left(F^{c}, \mu\right)$. We can prove that the generator ${ }_{F} L$ is selfadjoint in $L^{2}\left(F^{c}, \mu\right)$. Then ${ }_{F} P_{t}$ is reversible with respect to $\mu$. We also define the Dirichlet form

$$
\begin{aligned}
{ }_{F} D(f, f): & =-\left({ }_{F} L f, f\right) \\
& =\int_{F^{c}} \mu(d x) q(x) f^{2}(x)-\int_{F^{c} \times F^{c}} \mu(d x) q(x, d y) f(x) f(y) .
\end{aligned}
$$

Lemma 2.1 If there exist a decreasing function $\xi:[0, \infty) \rightarrow(0, \infty)$ such that $\xi(t) \rightarrow 0$ as $t \rightarrow \infty$ and $\mathbb{E}_{\mu} \xi\left(\tau_{F}\right)^{-1}=: c<\infty$, then WPID holds for $\alpha_{F}(r) \leq \xi^{-1}\left(\frac{r}{c}\right)$.

Proof

$$
\begin{aligned}
\left|\left(f,{ }_{F} P_{t} f\right)\right| & =\left|\int_{F^{c}} \mu(d x) f(x)_{F} P_{t} f(x)\right| \\
& \leq\|f\|_{L^{\infty}\left(F^{c}, \mu\right)}^{2} \int \mu(d x) \mathbb{P}_{x}\left[\tau_{F}>t\right] \\
& \leq\|f\|_{L^{\infty}\left(F^{c}, \mu\right)}^{2} \int \mu(d x) \mathbb{E}_{x} \xi\left(\tau_{F}\right)^{-1} \xi(t) \\
& =c\|f\|_{L^{\infty}\left(F^{c}, \mu\right)}^{2} \xi(t)
\end{aligned}
$$

where the last inequality holds since $\xi(t)$ is decreasing.

Since ${ }_{F} P_{t}$ is reversible with respect to $\mu$, by (2.2) we have

$$
\left\|_{F} P_{t / 2} f\right\|_{2}^{2} \leq c\|f\|_{L^{\infty}\left(F^{c}, \mu\right)}^{2} \xi(t) .
$$


Set $\eta(t)=c \xi(2 t)$. Then

$$
\left\|_{F} P_{t} f\right\|_{2}^{2} \leq\|f\|_{L^{\infty}\left(F^{c}, \mu\right)}^{2} \eta(t)
$$

By [9] this implies the nonergodic weak Poincaré inequality

$$
\|f\|_{L^{2}\left(F^{c}, \mu\right)}^{2} \leq{ }_{F} \alpha(r)_{F} D(f, f)+r\|f\|_{L^{\infty}\left(F^{c}, \mu\right)}^{2}, \quad r>0,
$$

for

$$
{ }_{F} \alpha(r) \leq 2 \eta^{-1}(r)=\xi^{-1}\left(\begin{array}{l}
r \\
c
\end{array}\right)
$$

For every $g$ with $\left.g\right|_{F}=0$, let $f=\left.g\right|_{F^{c}}$. Thus,

$$
\|f\|_{L^{2}\left(F^{c}, \mu\right)}=\|g\|_{2}, \quad\|f\|_{L^{\infty}\left(F^{c}, \mu\right)}=\|g\|_{\infty} .
$$

Besides, by (2.1) we have

$$
\begin{aligned}
D(g, g): & =-(L g, g) \\
& =\int_{E} \mu(d x) q(x) g^{2}(x)-\int_{E \times E} \mu(d x) q(x, d y) g(x) g(y) \\
& =\int_{F^{c}} \mu(d x) q(x) g^{2}(x)-\int_{F^{c} \times F^{c}} \mu(d x) q(x, d y) g(x) g(y) \\
& ={ }_{F} D(f, f) .
\end{aligned}
$$

By (2.3) it follows that

$$
\mu\left(g^{2}\right) \leq_{F} \alpha(r) D(g, g)+r\|g\|_{\infty}^{2}, \quad g \in \mathcal{D}(D),\left.g\right|_{F}=0, r>0 .
$$

Thus, (1.3) holds for $\alpha_{F}(r) \leq{ }_{F} \alpha(r) \leq \xi^{-1}\left(\frac{r}{c}\right)$.

\section{The relationship of WPID and WPI}

In this section, we show how to use the local Poincaré inequality and weak Poincaré inequality with absorbing boundary to obtain a weak Poincaré inequality.

\subsection{The relationship of WPID and WPI when $F$ is a singleton}

First, we consider the case that $F$ is a singleton. Assume that $F=\{\theta\}$ and $\mu(\theta)>0$. For convenience, we denote $\mu(\theta)=: \mu_{\theta}$ and $f(\theta)=: f_{\theta}$.

Lemma 3.1 For any $r>0$, define

$$
\alpha(r):=\inf \left\{C>0 \mid \operatorname{Var}_{\mu}(f) \leq C D(f, f)+r\|f\|_{\infty}^{2}, f \in \mathcal{D}(D)\right\}
$$

and

$$
\alpha_{\theta}(r):=\inf \left\{C_{\theta}>0 \mid \mu\left(f^{2}\right) \leq C_{\theta} D(f, f)+r\|f\|_{\infty}^{2}, f \in \mathcal{D}(D), f(\theta)=0\right\} .
$$


Then

$$
\alpha_{\theta}\left(\frac{r}{\mu_{\theta}}\right) \mu_{\theta} \leq \alpha(r) \leq \alpha_{\theta}\left(\frac{r}{4}\right)
$$

Proof (a) For the upper bound of $\alpha(r)$, noticing that $\operatorname{Var}_{\mu}(f)=\inf _{c} \mu\left((f-c)^{2}\right)$ and $\| f-$ $f_{\theta}\left\|_{\infty} \leq 2\right\| f \|_{\infty}$, for any $f \in \mathcal{D}(D)$, we have

$$
\begin{aligned}
\operatorname{Var}_{\mu}(f) & \leq \mu\left(\left(f-f_{\theta}\right)^{2}\right) \\
& \leq \alpha_{\theta}(r) D\left(f-f_{\theta}, f-f_{\theta}\right)+r\left\|f-f_{\theta}\right\|_{\infty}^{2} \\
& \leq \alpha_{\theta}(r) D(f, f)+4 r\|f\|_{\infty}^{2} .
\end{aligned}
$$

Then, by the definition of $\alpha(r)$ we have

$$
\alpha(r) \leq \alpha_{\theta}\left(\frac{r}{4}\right)
$$

(b) On the other hand, for any $f \in L^{2}(E)$ with $f_{\theta}=0$, we have

$$
\begin{aligned}
\operatorname{Var}_{\mu}(f) & =\mu\left(f^{2}\right)-\mu(f)^{2}=\mu\left(f^{2}\right)-\mu\left(f \mathbf{1}_{\{\theta\}}\right)^{2} \\
& \geq \mu\left(f^{2}\right)-\mu\left(f^{2}\right) \mu\left(\{\theta\}^{c}\right)=\mu\left(f^{2}\right) \mu_{\theta} .
\end{aligned}
$$

Thus,

$$
\mu\left(f^{2}\right) \mu_{\theta} \leq \operatorname{Var}_{\mu}(f) \leq \alpha(r) D(f, f)+r\|f\|_{\infty}^{2}
$$

and

$$
\mu\left(f^{2}\right) \leq \frac{1}{\mu_{\theta}} \alpha(r) D(f, f)+\frac{r}{\mu_{\theta}}\|f\|_{\infty}^{2} .
$$

Then, by the definition of $\alpha_{\theta}(r)$ we have

$$
\alpha_{\theta}\left(\frac{r}{\mu_{\theta}}\right) \leq \frac{1}{\mu_{\theta}} \alpha(r)
$$

Thus, by (3.2) and (3.3) we have $\alpha_{\theta}\left(\frac{r}{\mu_{\theta}}\right) \mu_{\theta} \leq \alpha(r) \leq \alpha_{\theta}\left(\frac{r}{4}\right)$.

\subsection{The relationship of WPI and WPID for general $F$}

For a general set $F$, we consider the relationships of $\alpha(r)$ and $\alpha_{F}(r)$ by the decomposition method and the conclusions given in Section 3.1.

Thinking of $F$ as a point $\theta$, we construct another state space $\hat{E}$ and $q$-process with $q$-pair $(\hat{q}(x), \hat{q}(x, d y))$ as follows: $\hat{E}=\{\theta\} \cup F^{c}$ and $\hat{\mathscr{E}}=\sigma\left(\{\theta\}, \mathscr{E} \cap F^{c}\right)$. For any $x \in F^{c}$ and $H \in \hat{\mathscr{E}}$, define

$$
\hat{q}(x, H)= \begin{cases}q(x, F)+q(x, H \backslash\{\theta\}), & \theta \in H, \\ q(x, H), & \theta \notin H,\end{cases}
$$


and, for $x=\theta$ and $H \in \hat{\mathscr{E}}$,

$$
\hat{q}(\theta, H)=\frac{1}{\mu(F)} \int_{F} \mu(d y) q(y, H \backslash\{\theta\}),
$$

and, for $x \in E, \hat{q}(x)=\hat{q}(x, \hat{E})$. Then $(\hat{q}(x), \hat{q}(x, d y))$ is a reversible $q$-pair on $(\hat{E}, \hat{\mathscr{E}})$ with respect to the probability measure $\hat{\mu}$, where, for any $H \in \hat{\mathscr{E}}$,

$$
\hat{\mu}(H)= \begin{cases}\mu(F)+\mu(H \backslash\{\theta\}), & \theta \in H, \\ \mu(H), & \theta \notin H .\end{cases}
$$

Denote

$$
\hat{\alpha}_{\theta}(r):=\inf \left\{\hat{C}_{\theta} \mid \hat{\mu}\left(\hat{f}^{2}\right) \leq \hat{C}_{\theta} \hat{D}(\hat{f}, \hat{f})+r\|\hat{f}\|_{\infty}^{2}, \hat{f} \in \mathcal{D}(\hat{D}), \hat{f}(\theta)=0\right\}
$$

and

$$
\hat{\alpha}(r):=\inf \left\{\hat{C} \mid \operatorname{Var} \mu(\hat{f}) \leq \hat{C} \hat{D}(\hat{f}, \hat{f})+r\|\hat{f}\|_{\infty}^{2}, \hat{f} \in \mathcal{D}(\hat{D})\right\}
$$

where $\hat{D}(\hat{f}, \hat{f})=\frac{1}{2} \int_{\hat{E} \times \hat{E}} \hat{\mu}(d x) \hat{q}(x, d y)(\hat{f}(y)-\hat{f}(x))^{2}$ is the Dirichlet form of the $q$-pair $(\hat{q}(x)$, $\hat{q}(x, d y))$ in $L^{2}(\hat{E}, \hat{\mathscr{E}})$.

It is easy to see that $\hat{\alpha}_{\theta}(r)=\alpha_{F}(r)$. By Lemma 3.1 we have a nice relationship $\hat{\alpha}_{\theta}\left(\frac{r}{\hat{\mu}_{\theta}}\right) \hat{\mu}_{\theta} \leq$ $\hat{\alpha}(r) \leq \hat{\alpha}_{\theta}\left(\frac{r}{4}\right)$. So we obtain the following lemma.

\section{Lemma 3.2}

$$
\alpha_{F}\left(\frac{r}{\mu(F)}\right) \mu(F) \leq \hat{\alpha}(r) \leq \alpha_{F}\left(\frac{r}{4}\right) .
$$

Next, we first prove the relationships between $\hat{\alpha}(r)$ and $\alpha(r)$.

\section{Lemma 3.3}

$$
\hat{\alpha}(r) \leq \alpha(r) \leq\left(1+\frac{M_{F}}{\lambda_{1}(F)}\right) \hat{\alpha}(r)+\frac{1}{\lambda_{1}(F)} .
$$

Proof (a) For the lower bound, given an arbitrary function $\hat{f}$ on $\hat{E}$, set

$$
f(x)= \begin{cases}\hat{f}(\theta), & x \in F, \\ \hat{f}(x), & x \in F^{c}\end{cases}
$$

By the definition of $\alpha(r)$, for this function $f$, we have

$$
\operatorname{Var}_{\mu}(f) \leq \alpha(r) D(f, f)+r\|f\|_{L^{\infty}(E, \mu)}^{2}
$$

By a simple calculation we get $\mu(f)=\hat{\mu}(\hat{f}), \mu\left(f^{2}\right)=\hat{\mu}\left(\hat{f}^{2}\right)$, and $D(f, f)=\hat{D}(\hat{f}, \hat{f})$. Noticing that $\|f\|_{L^{\infty}(E, \mu)}=\|\hat{f}\|_{L^{\infty}(\hat{E}, \hat{\mu})}$, we have

$$
\operatorname{Var}_{\hat{\mu}}(\hat{f}) \leq \alpha(r) \hat{D}(\hat{f}, \hat{f})+r\|\hat{f}\|_{L^{\infty}(\hat{E}, \hat{\mu})}^{2}
$$


We obtain

$$
\hat{\alpha}(r) \leq \alpha(r)
$$

(b) On the other hand, for any function $f$ on $E$, we set

$$
g(x)= \begin{cases}\mu_{F}(f), & x \in F \\ f(x), & x \in F^{c}\end{cases}
$$

and

$$
\hat{f}(x)= \begin{cases}\mu_{F}(f), & x=\theta \\ f(x), & x \in F^{c}\end{cases}
$$

It is easy to see that $\mu(g)=\hat{\mu}(\hat{f})=\mu(f)$. Thus, we have

$$
\begin{aligned}
\operatorname{Var}_{\mu}(f)= & \mu\left((f-\mu(f))^{2}\right) \\
= & \mu\left((f-g)^{2}\right)+\mu\left((g-\mu(f))^{2}\right) \\
= & \mu(F) \operatorname{Var}_{\mu_{F}}(f)+\operatorname{Var}_{\hat{\mu}}(\hat{f}) \\
\leq & \frac{\mu(F)}{\lambda_{1}(F)} D_{F}(f, f)+\hat{\alpha}(r) \hat{D}(\hat{f}, \hat{f})+r\|\hat{f}\|_{L^{\infty}(\hat{E}, \hat{\mu})}^{2} \\
= & \frac{1}{2 \lambda_{1}(F)} \int_{F \times F} \mu(d x) q(x, d y)(f(y)-f(x))^{2} \\
& +\hat{\alpha}(r)\left(\int_{F^{c}} \mu(d x) q(x, F)\left(f(x)-\mu_{F}(f)\right)^{2}\right. \\
& \left.+\frac{1}{2} \int_{F^{c} \times F^{c}} \mu(d x) q(x, d y)(f(y)-f(x))^{2}\right)+r\|f\|_{L^{\infty}(E, \mu)}^{2} .
\end{aligned}
$$

For each $x \in F^{c}$ with $q(x, F)>0$, we set

$$
\mu_{F}^{x}(d y)=\frac{q(x, d y)}{q(x, F)} .
$$

Let $1 \leq p, q \leq \infty$ such that $\frac{1}{p}+\frac{1}{q}=1$ to be determined later. Then we have

$$
\begin{aligned}
& \int_{F^{c}} \mu(d x) q(x, F)\left(f(x)-\mu_{F}(f)\right)^{2} \\
& \leq \int_{F^{c}} \mu(d x) q(x, F)\left[p\left(f(x)-\mu_{F}^{x}(f)\right)^{2}+q\left(\mu_{F}^{x}(f)-\mu_{F}(f)\right)^{2}\right] \\
& \leq \int_{F^{c}} \mu(d x) q(x, F)\left[p \int_{F} \mu_{F}^{x}(d y)(f(x)-f(y))^{2}+q \int_{F} \mu_{F}^{x}(d y)\left(f(y)-\mu_{F}(f)\right)^{2}\right] \\
& \leq p \int_{F \times F^{c}} \mu(d x) q(x, d y)(f(y)-f(x))^{2} \\
& \quad+\frac{q M_{F}}{2 \lambda_{1}(F)} \int_{F \times F} \mu(d x) q(x, d y)(f(y)-f(x))^{2} .
\end{aligned}
$$


Combining (3.7) and (3.8), we have

$$
\begin{aligned}
\operatorname{Var}_{\mu}(f) \leq & \frac{1}{2 \lambda_{1}(F)} \int_{F \times F} \mu(d x) q(x, d y)(f(y)-f(x))^{2} \\
& +\hat{\alpha}(r)\left\{\frac{1}{2} \int_{F^{c} \times F^{c}} \mu(d x) q(x, d y)(f(y)-f(x))^{2}\right. \\
& +p \int_{F \times F^{c}} \mu(d x) q(x, d y)(f(y)-f(x))^{2} \\
& \left.+\frac{q M_{F}}{2 \lambda_{1}(F)} \int_{F \times F} \mu(d x) q(x, d y)(f(y)-f(x))^{2}\right\}+r\|f\|_{\infty}^{2} \\
\leq & \max \left\{p \hat{\alpha}(r), \frac{1+q M_{F} \hat{\alpha}(r)}{\lambda_{1}(F)}\right\} D(f, f)+r\|f\|_{\infty}^{2} .
\end{aligned}
$$

Recall that $p^{-1}+q^{-1}=1$. Let $p_{0}$ be the solution to the equation

$$
p \hat{\alpha}(r)=\frac{1+\frac{p}{p-1} M_{F} \hat{\alpha}(r)}{\lambda_{1}(F)}
$$

Then we have

$$
p_{0}=\frac{\left(\lambda_{1}(F)+M_{F}\right) \hat{\alpha}(r)+1+\sqrt{\left(\left(\lambda_{1}(F)+M_{F}\right) \hat{\alpha}(r)+1\right)^{2}-4 \lambda_{1}(F) \hat{\alpha}(r)}}{2 \lambda_{1}(F) \hat{\alpha}(r)} .
$$

So we obtain $\operatorname{Var}_{\mu}(f) \leq \alpha(r) D(f, f)+r\|f\|_{\infty}^{2}, f \in \mathcal{D}(D), r>0$, where

$$
\begin{aligned}
\alpha(r) & \leq p_{0} \hat{\alpha}(r) \\
& =\frac{\left(\lambda_{1}(F)+M_{F}\right) \hat{\alpha}(r)+1+\sqrt{\left(\left(\lambda_{1}(F)+M_{F}\right) \hat{\alpha}(r)+1\right)^{2}-4 \lambda_{1}(F) \hat{\alpha}(r)}}{2 \lambda_{1}(F)} \\
& \leq \frac{\left(\lambda_{1}(F)+M_{F}\right) \hat{\alpha}(r)+1}{\lambda_{1}(F)} .
\end{aligned}
$$

Combining (3.6) and (3.9), we have

$$
\hat{\alpha}(r) \leq \alpha(r) \leq\left(1+\frac{M_{F}}{\lambda_{1}(F)}\right) \hat{\alpha}(r)+\frac{1}{\lambda_{1}(F)}
$$

So by (3.4) we get

$$
\alpha_{F}\left(\frac{r}{\mu(F)}\right) \mu(F) \leq \alpha(r) \leq\left(1+\frac{M_{F}}{\lambda_{1}(F)}\right) \alpha_{F}\left(\frac{r}{4}\right)+\frac{1}{\lambda_{1}(F)}
$$

Recall that by Lemma 2.1 we have $\alpha_{F}(r) \leq \xi^{-1}\left(\frac{r}{c}\right)$. Then Theorem 1.1 follows from Lemma 2.1 .

The authors declare that they have no competing interests. 


\section{Author details}

${ }^{1}$ College of Mathematics and Information Sciences, North China University of Water Resources and Electric Power,

Zhengzhou, 450045, P.R. China. ${ }^{2}$ School of Mathematics and Statistics, Henan University, Kaifeng, 475004, P.R. China.

\section{Acknowledgements}

The authors would like to thank the referees for their careful reading and constructive comments. This study was partially supported by the NSFC (Nos. 11201145; 11526075).

Received: 8 April 2016 Accepted: 20 July 2016 Published online: 03 August 2016

\section{References}

1. Röckner, M, Wang, FY: Weak Poincaré inequalities and $L^{2}$-convergence rates of Markov semigroups. J. Funct. Anal. 185(2), 564-603 (2001)

2. Barkry, D, Cattiaux, P, Guillin, A: Rate of convergence for ergodic continuous Markov processes: Lyapunov versus Poincaré. J. Funct. Anal. 254, 727-759 (2008)

3. Cattiaux, P, Guillin, A, Wang, FY, Wu, LM: Lyapunov conditions for super Poincaré inequality. J. Funct. Anal. 256 , 1821-1841 (2009)

4. Cattlaux, P, Guillin, A, Zitt, PA: Poincaré inequalities and hitting times. Ann. Inst. Henri Poincaré Probab. Stat. 49(1), 95-118 (2013)

5. Mao, YH, Xia, XH: Spectral gap for jump processes by decomposition method. Front. Math. China 4(2), 335-348 (2009)

6. Cheng, LJ, Wang, YZ: Algebraic convergence rate for reflecting diffusion processes on manifolds with boundary. Potential Anal. 44, 91-107 (2016)

7. Wang, FY: Functional Inequalities, Markov Semigroups and Spectral Theory. Science Press, Beijing (2005)

8. Chen, MF: From Markov Chains to Non-equilibrium Partical Systems. World Scientific, Beijing (2004)

9. Wang, FY: Functional inequalities for the decay of sub-Markov semigroups. Potential Anal. 18, 1-23 (2003)

\section{Submit your manuscript to a SpringerOpen ${ }^{\circ}$ journal and benefit from:}

- Convenient online submission

Rigorous peer review

- Immediate publication on acceptance

- Open access: articles freely available online

- High visibility within the field

- Retaining the copyright to your article 